\title{
Accesibilidad al entorno físico en instalaciones de acondicionamiento para personas con discapacidad física: una revisión integradora
}

Accessibility in Fitness Facilities for People with Physical Disabilities: An Integrative Review Acessibilidade ao ambiente físico em academias de ginástica para pessoas com deficiência física: uma revisão integrativa

Iván Darío Ortega Luna, FT*

María Angélica Ortiz Hernández, FT²

Carlos Martín Cervantes Olivares, EF, MA y PhD³

Luis Fernando Rodríguez Ibagué, FT y MSc. ${ }^{1}$

Recibido: 3 de marzo de 2020 · Aceptado: 8 de septiembre de 2020

Doi: https://doi.org/10.12804/revistas.urosario.edu.co/revsalud/a.10151

Para citar este artículo: Ortega Luna ID, Ortiz Hernández MA, Cervantes Olivares CM, Rodríguez Ibagué LF. Accesibilidad al entorno físico en instalaciones de acondicionamineto para personas con discapacidad física: una revisión integradora. Rev Cienc Salud. 2021;19(1):1-21. https://doi.org/10.12804/revistas.urosario.edu.co/revsalud/a.10151

1 Universidad del Rosario, Escuela de Medicina y Ciencias de la Salud, Programa de Fisioterapia, Línea de profundización en Administración y Emprendimiento en Rehabilitación (Colombia).

2 Universidad del Rosario, Escuela de Medicina y Ciencias de la Salud, Programa de Fisioterapia, Línea de profundización en Actividad Física y Salud (Colombia).

3 Universidad Huston-Tillotson, Escuela de Artes y Ciencias, Departamento de Kinesiología, Educación Física Adaptada (Estados Unidos).

Iván Dario Ortega Luna: ORCID https://orcid.org/0000-0001-6934-6286

María Angélica Ortiz Hernández: orciD https://orcid.org/0000-0002-6058-9246

Carlos Martín Cervantes Olivares: ORCiD https://orcid.org/0000-0001-7626-5687

Luis Fernando Rodríguez Ibagué: ORCID https://orcid.org/0000-0001-6129-8689

Autor de correspondencia: ivan.ortega@urosario.edu.co 


\section{Resumen}

Introducción: las personas en condición de discapacidad están expuestas a diferentes barreras físicas o arquitectónicas que impiden su acceso a instalaciones y su participación en actividades físicas, ejercicio y deporte. Esto les dificulta obtener los beneficios que implica su práctica y conduce a un deterioro de su calidad de vida. Materiales y métodos: revisión integradora de estudios cuantitativos a través de la metodología SPIDER, con los siguientes componentes: 1) identificación del problema, 2) búsqueda de literatura, 3) evaluación de datos y 4) análisis de estos. Resultados: se incluyeron 7 estudios en los cuales se evaluaron 429 instalaciones de acondicionamiento con 4 instrumentos de medición, por medio de la puntuación media en porcentajes identificados por el tipo de accesibilidad al entorno físico (al desplazamiento y a la implementación deportiva y recreativa), donde el $0 \%$ es la accesibilidad mínima y el $100 \%$ es la accesibilidad máxima. En ninguno de los estudios incluidos se identificó una media mayor al 60\%. La accesibilidad a la implementación deportiva y recreativa fue la más baja, con el 19.81\%, y la accesibilidad física fue la más alta, con el 57.01\%. Conclusión: las instalaciones de acondicionamiento no son del todo accesibles para personas con discapacidad. Existe la gran necesidad de investigación en la accesibilidad a la implementación deportiva y recreativa en Latinoamérica y fundamentalmente en Colombia.

Palabras clave: personas con discapacidad; accesibilidad a los servicios de salud; accesibilidad arquitectónica; centros de acondicionamiento; instalaciones deportivas y recreativas.

\section{Abstract}

Introduction: People with disabilities are often exposed to different physical or architectural barriers that block access to their participation in physical activity, exercise, and sports. This makes it difficult for them to benefit from these facilities which lead to a deterioration in the quality of life for many individuals with disabilities. Materials and Methods: An integrative review of quantitative studies was conducted using the SPIDER methodology with the following components: (i) problem identification, (ii) literature search, (iii) data evaluation, and (iv) data analysis. Results: Seven studies were included in which 429 fitness facilities were evaluated with four measuring instruments including mean ratings in percentages identified by the type of accessibility (physical, displacement, sports and recreational implementation) and whether they were at $0 \%$ minimum accessibility or at $100 \%$ maximum accessibility. None of the studies that were included obtained a mean score greater than $60 \%$. Accessibility to sports and recreational implementation was at the lowest with $19.81 \%$ and physical accessibility was at the highest with 57.01\%. Conclusion: Fitness facilities are not entirely accessible for people with disabilities. There is a need for further research to look into accessibility of sports and recreational implementation in Latin America, especially in Colombia.

Keywords: People with disabilities; health services accessibility; architectural accessibility; fitness centers; sports and recreational facilities.

\section{Resumo}

Introdução: as pessoas deficientes estão expostas a diferentes barreiras físicas ou arquitetônicas que impedem seu acesso às instalações e participação em atividade física, exercício e esporte, dificultando obter o benefício que a prática de esportes traz, o que prejudica sua qualidade de vida. Materiais $e$ métodos: realizou-se uma revisão integrativa de estudos quantitativos através da metodologia SPIDER com os seguintes componentes: 1) identificação do problema, 2) revisão de literatura, 3) avaliação dos dados e 4) análise de dados. Resultados: foram incluídos sete estudos, nos quais se avaliaram um total de 429 academias com quatro instrumentos de medição, por meio da pontuação média em porcentagem identificada pelo tipo de acesso ao ambiente físico (física, por deslocamento, e aos implementos esportivos e recreativos), sendo 0\% acessibilidade mínima e 100\% acessibilidade máxima. Em nenhum dos estudos incluídos identificou-se uma média superior a 60\%. A acessibilidade aos implementos esportivos e recreativos foi a mais baixa com $19,81 \%$ e a acessibilidade física foi a mais alta com 57,01\%. Conclusão: as instalações das academias não são completamente acessíveis para pessoas com deficiência. Existe 
uma grande necessidade de investigação, principalmente na acessibilidade de implementos esportivos e recreativos na América Latina e fundamentalmente, na Colômbia.

Palavras-chave: pessoas com deficiência; acessibilidade aos serviços de saúde; acessibilidade arquitetônica; centros de treinamento; instalações esportivas e recreativas.

\section{Introducción}

工 a Organización Mundial de la Salud ha estimado que más de mil millones de personas al $15 \%$ de la población total, y ha proyectado que estas cifras seguirán en incremento, debido al envejecimiento y al aumento de la prevalencia de las enfermedades crónicas (1). En Colombia, un censo del 2005 del Departamento Administrativo Nacional de Estadística encontró una prevalencia del $6.4 \%$, que corresponde a 2624898 personas en condición de discapacidad, quienes, en su mayoría, tienen discapacidades de tipo motor y visual; mientras que los resultados preliminares del Censo del 2018 muestran que el 7.2\% de la población presenta alguna dificultad funcional para realizar sus actividades diarias (2-4). Por otro lado, a partir del 2002, Colombia cuenta con el Registro para la Localización y Caracterización de Personas con Discapacidad, el cual, pese a que posee un conjunto de variables que permite observar desde un panorama más amplio la situación actual, tiene una limitada cobertura poblacional con el registro, en junio del 2018, de 1404108 personas en condición de discapacidad, lo que equivale al $2.6 \%$ de la población colombiana (5). La investigación científica sugiere que en el colectivo de las personas en condición de discapacidad física hay mayores índices de sedentarismo, lo cual conduce al deterioro del estado de salud, a limitaciones de la actividad, a restricciones para la participación y a una menor calidad de vida, debido a su propia discapacidad, a las barreras sociales y a las barreras físicas o arquitectónicas que impiden el acceso a las instalaciones y a la participación de actividad física y deporte $(1,6)$.

La actividad física y el deporte son estrategias ideales para: 1) la promoción de la salud, el bienestar y la inclusión social; 2) la prevención de problemas de salud relacionados con el estilo de vida (enfermedades cardiacas, cáncer y diabetes), y 3) la disminución de los riesgos secundarios a la condición de discapacidad primaria (úlceras por presión, desacondicionamiento físico y depresión) (7,8). Generalmente, las personas con discapacidad no obtienen la misma cantidad de actividad física y oportunidades atléticas en comparación con el resto de la población, debido a que muchos centros de acondicionamiento, espacios al aire libre y algunos tipos de equipamiento no son accesibles y dificultan los esfuerzos para su participación (7). Las necesidades de actividad física y ejercicio de las personas en condición de discapacidad son similares a las del resto de la población, ya que requieren optimizar la 
capacidad aeróbica, fuerza y resistencia, movilidad y flexibilidad para mejorar/mantener su funcionalidad (9). De acuerdo con el Departamento de Salud y Servicios Humanos de Estados Unidos, los adultos con discapacidad deben realizar actividad aeróbica de intensidad moderada, por lo menos, durante 150 minutos a la semana o actividad aeróbica de intensidad vigorosa durante, por lo menos, 75 minutos a la semana, complementada con el entrenamiento de resistencia de intensidad moderada o alta, dos o más días a la semana en todos los grupos musculares principales (10).

En Estados Unidos, el título III del Acta de Estadounidenses con Discapacidades (ADA, por sus siglas en inglés) establece los requisitos de diseño para la construcción y adecuación de instalaciones aplicadas a lugares de alojamiento público, instalaciones comerciales e instalaciones gubernamentales estatales y locales (11). En Colombia, el Censo de Escenarios Deportivos y Recreativos permitió conocer la ubicación, el estado, las dimensiones y la capacidad de estos, y reveló datos preocupantes sobre la adecuación de la infraestructura para personas con discapacidad (12). Esta situación llevó a que el Departamento Administrativo del Deporte, la Recreación, la Actividad Física y el Aprovechamiento del Tiempo Libre (Coldeportes) y el Comité Paralímpico Colombiano reconocieran la importancia del Estado y de los entes territoriales en generar inclusión social y atender las realidades desde el ámbito deportivo y recreativo a poblaciones vulnerables (13). En consecuencia, en el 2009 se redactó la Guía de diseño accesible y universal, la cual ofrece los requerimientos legales y técnicos de los espacios accesibles para las personas con discapacidad en los diferentes escenarios deportivos (13). En el 2013, la Norma Técnica Colombiana de Accesibilidad 6047 estableció los criterios y requisitos generales de accesibilidad y señalización para los espacios de acceso al ciudadano, con la creación de una herramienta de autodiagnóstico de espacios físicos que recoge y sintetiza en una lista de verificación aspectos relacionados con espacios exteriores e interiores, itinerarios, acceso, mobiliario, señalización, entre otros (14).

Por lo visto, existen normas internacionales y nacionales que garantizan el acceso al entorno construido para personas con discapacidades $(11,14)$. Sin embargo, aunque la Norma Técnica Colombiana de Accesibilidad 6047 abarca bastantes aspectos relacionados con el entorno físico, no contempla la accesibilidad específicamente a instalaciones de acondicionamiento físico (14). En el 2013, Coldeportes definió cinco Lineamientos para el fomento y la inclusión en la práctica de educación física, recreación, actividad física y deporte dirigidas a las personas con discapacidad, a partir de la clasificación de la accesibilidad: 1) comunicación e información, 2) calidad de servicios y programas, 3) formación académica, 4) simbólica (transformación de imaginarios) y 5) entorno físico (15). Esta investigación se enfoca especialmente en el último lineamiento, el cual se define como "un buen diseño incluyente para todas las personas, indispensables para la equiparación de oportunidades de personas con discapacidad en el ámbito recreativo o deportivo" (15). El lineamiento contempla tres puntos clave: 
- Accesibilidadfísica: identificación y eliminación de obstáculos y barreras físicas en teléfonos públicos, baños, escaleras, semáforos, vestuarios, entre otras (15).

- Accesibilidad al desplazamiento: relacionada con la movilidad humana con la mayor independencia posible (medios de transporte, desplazamiento a sitios de práctica deportiva y recreativa) (15).

- Accesibilidad en implementación deportiva y recreativa: implementos de buena calidad (costo/beneficio) y que sean adaptados a necesidades específicas si así lo requiere (15).

Por lo anterior, es necesario identificar los obstáculos y las desigualdades en la participación de la actividad física, el ejercicio y el deporte para superar las barreras de accesibilidad al entorno físico que enfrentan día a día las personas con discapacidad física y, de esta manera, aportar a las recomendaciones de la Organización Mundial de la Salud en el sentido de recopilar datos sobre discapacidad, así como de reforzar y apoyar la investigación sobre el tema (1). El propósito de este estudio fue sintetizar críticamente la literatura sobre la accesibilidad al entorno físico para personas con discapacidad física en instalaciones de acondicionamiento. Esta investigación también buscó: 1) clasificar proporcionalmente el tipo de práctica y el tipo de accesibilidad al entorno físico; 2) describir las características de las instalaciones; 3) identificar los instrumentos de medición y los resultados de accesibilidad al entorno físico para personas con discapacidad física en instalaciones de acondicionamiento físico, y 4) analizar la literatura según los tipos de accesibilidad al entorno físico en instalaciones de acondicionamiento físico.

\section{Materiales y métodos}

$\mathrm{E}$ vos con la intención de examinar los resultados y las relaciones entre variables de metodologías y propuestas que se hayan realizado previamente (17). Para el desarrollo de este tipo de revisión se deben tener en cuenta los siguientes pasos: la identificación del problema, la búsqueda de literatura, la evaluación de los datos y el análisis de los datos (reducción, visualización y comparación) $(18,19)$.

Identificación del problema. El problema se abordó por medio de la metodología SPIDER, en la cual: S (sample) es muestra: instalaciones de acondicionamiento físico; PI (phenomenon of interest) es fenómeno de interés: accesibilidad al entorno físico (física, al desplazamiento y a la implementación deportiva y recreativa) para personas con discapacidad física, según la categorización de la Resolución 00583 de 2018, emitida por el Ministerio de Salud y Protección 
Social de Colombia (20); D (design) es diseño: instrumento de medición; E (evaluation) es evaluación: examinación y resultados de accesibilidad al entorno físico; y R (research type) es tipo de investigación: cuantitativo (21).

Búsqueda de la literatura. Dos investigadores realizaron la búsqueda de literatura. Los filtros de esta revisión fueron fuentes primarias de publicaciones académicas indexadas y publicadas entre enero del 2003 y diciembre del 2018, en inglés y español, con acceso a texto completo y disponibles en bases de datos electrónicas con título, resumen o palabras clave relacionados con la accesibilidad al entorno físico para personas con discapacidad física en instalaciones de acondicionamiento físico (22). La búsqueda digital de base de datos científicas se llevó a cabo en Medline, Academic Search Complete, Complementary Index, Scopus, Science Direct, Public Health Database ProQuest y uILAcs. Se emplearon los descriptores del vocabulario controlado según los encabezados de materia médica con sus siglas en inglés (MeSH): Physical Disability AND Architectural Accessibility or Facility Access AND Fitness Centers; y sinónimos asociados en inglés: people with physical disabilities, person with physical disabilities, people with physical disability, person with physical disability, AND architectural accessibility, physical barriers, facility access, health services accessibility AND fitness center, wellness center, health club, Gym; y con los descriptores controlados de las ciencias de la salud (DeCS) en español: personas con discapacidad física $\mathrm{Y}$ estructuras de acceso $\mathrm{O}$ barreras físicas $\mathrm{O}$ barreras arquitectónicas $\mathrm{O}$ acceso a las instalaciones $\mathrm{Y}$ centros de acondicionamiento $\mathrm{O}$ gimnasios; y sinónimos asociados en español: personas con discapacidades físicas, personas con discapacidad física, persona con discapacidad física, persona con discapacidades físicas, Y estructuras de acceso, barreras arquitectónicas, barreras físicas, acceso a las instalaciones, accesibilidad a los servicios de salud Y centro de acondicionamiento, club de salud, centro de bienestar, gimnasio.

Evaluación de datos. Después de la búsqueda de fuentes primarias, dos investigadores de manera independiente efectuaron un cribado inicial con el título; posteriormente, se revisaron el resumen y las palabras clave relacionadas. Un tercer investigador evaluó los cribados de manera independiente para corroborar los resultados e incluir los estudios. Si se encontraba algún desacuerdo entre los investigadores con respecto a un artículo, se discutía hasta llegar a un acuerdo. Los criterios de inclusión fueron: a) estudios publicados en revista académica; b) estudios cuantitativos; c) estudios que hubieran evaluado uno o más tipos de accesibilidad al entorno físico (accesibilidad física; accesibilidad al desplazamiento o accesibilidad en implementación deportiva y recreativa) en instalaciones de acondicionamiento físico; d) población objetivo de personas con discapacidad física según la Resolución 00583 de 2018, emitida por el Ministerio de Salud y Protección Social de Colombia (20); e) estudios que contengan muestra de instalaciones de acondicionamiento físico; y f) estudios que reporten instrumento de medición y resultados. Los criterios de exclusión fueron investigaciones que no cumplían con los criterios de inclusión, y que no fueran publicados en su totalidad (19,21). El proceso de selección se puede observar en la figura 1. 
Fuentes encontradas en inglés y español:

- Medline: $\mathrm{n}=153$

- Academic Search Complete: $\mathrm{n}=205$

- Complementary Index: $n=148$

- Scopus: $\mathrm{n}=11$

- Science Direct: $\mathrm{n}=19$

- ProQuest: $\mathrm{n}=45$

- Lilacs: $\mathrm{n}=13$

Total: $\mathrm{n}=594$

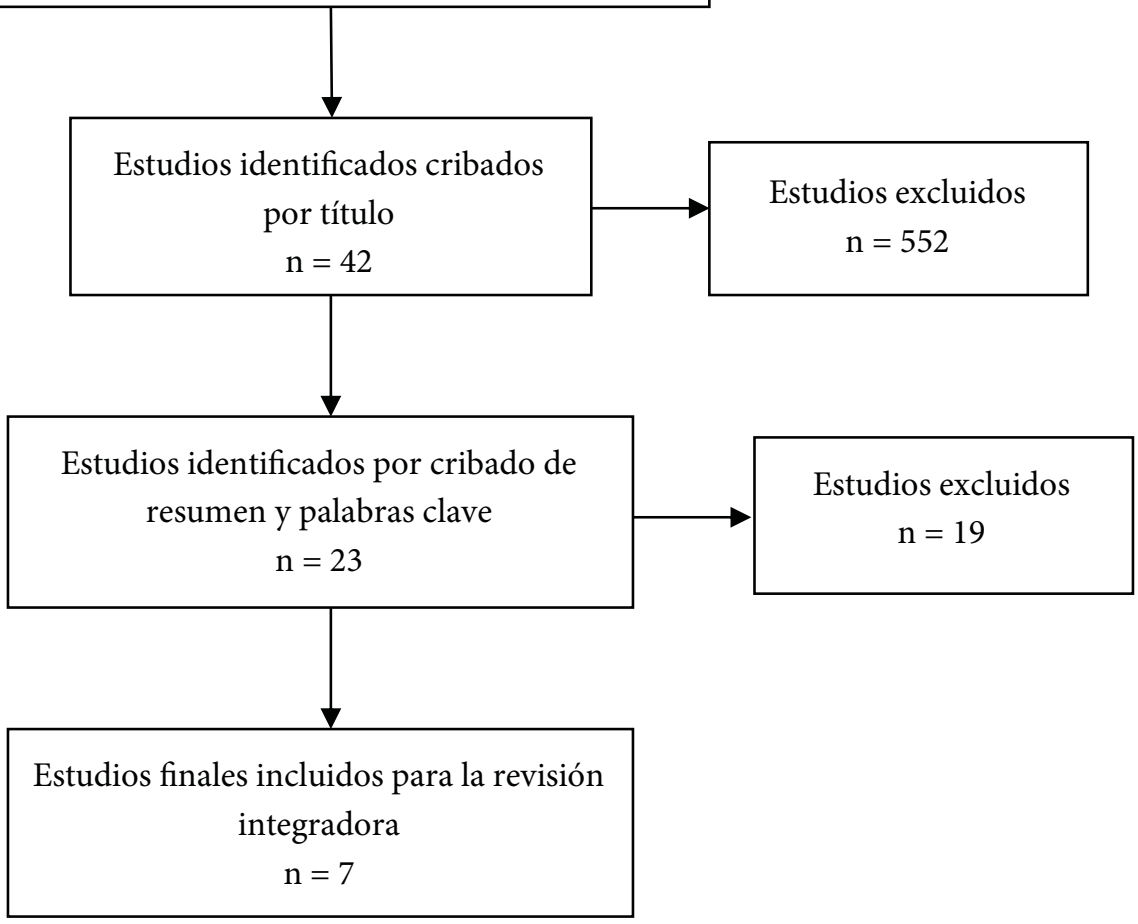

Figura 1. Proceso de selección de estudios

Análisis de los datos (extracción, visualización y análisis). En la última etapa de la revisión se utilizó una matriz para la extracción y visualización de los datos (tabla 1), que contiene: referencia, país de origen, número y características de la muestra (instalaciones de acondicionamiento), tipo de práctica, instrumento de medición, identificación del tipo de accesibilidad al entorno físico (dominios o secciones de evaluación seleccionadas) $(22,23)$. Dos investigadores realizaron de manera independiente la extracción de datos y un tercer investigador los revisó, con el fin de obtener resultados consistentes. Posteriormente, se realizó un análisis descriptivo de los datos. 
Tabla 1. Matriz de extracción y visualización de datos

\begin{tabular}{|c|c|c|c|c|c|c|}
\hline n. ${ }^{\circ}$ & Referencia & $\begin{array}{l}\text { País de } \\
\text { origen }\end{array}$ & $\begin{array}{c}\text { Número y } \\
\text { características } \\
\text { de la muestra } \\
\text { (instalaciones de } \\
\text { acondicionamiento) }\end{array}$ & $\begin{array}{l}\text { Tipo de } \\
\text { práctica }\end{array}$ & $\begin{array}{l}\text { Instrumento } \\
\text { de medición }\end{array}$ & $\begin{array}{l}\text { Identificación del } \\
\text { tipo y descripción de } \\
\text { accesibilidad al entorno } \\
\text { físico (dominios/ } \\
\text { secciones de evaluación } \\
\text { seleccionadas) }\end{array}$ \\
\hline 1 & $\begin{array}{l}\text { Arbour y } \\
\text { Ginis, } 2011 \\
(25)\end{array}$ & Canadá & $\begin{array}{l}44 \text { instalaciones re- } \\
\text { creativas y centros } \\
\text { de acondicionamien- } \\
\text { to físico. }\end{array}$ & $\begin{array}{l}\text { Actividad } \\
\text { física } \\
\text { Ejercicio }\end{array}$ & $\begin{array}{l}\text { Versión modi- } \\
\text { ficada del Ins- } \\
\text { trumento de } \\
\text { Accesibilidad } \\
\text { para la Me- } \\
\text { dición de En- } \\
\text { tornos de Re- } \\
\text { creación y de } \\
\text { Acondiciona- } \\
\text { miento Físico } \\
\text { con } 10 \text { de las } \\
16 \text { subescalas } \\
\text { originales. }\end{array}$ & $\begin{array}{l}\text { Accesibilidad física: áreas } \\
\text { de entrada, baños, vestua- } \\
\text { rios, ascensores. } \\
\text { Accesibilidad al desplaza- } \\
\text { miento: estacionamiento. } \\
\text { Accesibilidad en imple- } \\
\text { mentación deportiva y re- } \\
\text { creativa: equipamiento. }\end{array}$ \\
\hline 2 & $\begin{array}{l}\text { Sá et al., } \\
2012(26)\end{array}$ & Portugal & $\begin{array}{l}11 \text { complejos-insta- } \\
\text { laciones deportivas } \\
\text { públicas. }\end{array}$ & Deporte & $\begin{array}{l}\text { Creación de } \\
\text { lista de verifi- } \\
\text { cación basada } \\
\text { en las normas } \\
\text { y la legislación } \\
\text { portuguesa y } \\
\text { europea }\end{array}$ & $\begin{array}{l}\text { Accesibilidad física: acce- } \\
\text { so externo, rampas, escale- } \\
\text { ras, acceso interior, ascen- } \\
\text { sor, circulación interior, } \\
\text { instalaciones sanitarias } \\
\text { (duchas y vestuarios), ins- } \\
\text { talación eléctrica. }\end{array}$ \\
\hline 3 & $\begin{array}{l}\text { Rimmer } \\
\text { et al., } \\
2017 \text { (27) }\end{array}$ & $\begin{array}{l}\text { Estados } \\
\text { Unidos }\end{array}$ & $\begin{array}{l}227 \text { centros de acon- } \\
\text { dicionamiento físico. }\end{array}$ & $\begin{array}{l}\text { Actividad } \\
\text { física } \\
\text { Ejercicio }\end{array}$ & $\begin{array}{l}\text { Instrumento } \\
\text { de Accesibi- } \\
\text { lidad para la } \\
\text { Medición de } \\
\text { Entornos de } \\
\text { Recreación y } \\
\text { de Acondicio- } \\
\text { namiento Fí- } \\
\text { sico. }\end{array}$ & $\begin{array}{l}\text { Accesibilidad física: rutas } \\
\text { de acceso y áreas de entra- } \\
\text { da, vestuarios y duchas, } \\
\text { spas (tinas de hidromasaje, } \\
\text { bañeras de hidromasaje, } \\
\text { saunas, salas de vapor), ba- } \\
\text { ños, fuentes de agua. } \\
\text { Accesibilidad al desplaza- } \\
\text { miento: estacionamiento. } \\
\text { Accesibilidad en imple- } \\
\text { mentación deportiva y re- } \\
\text { creativa: equipamiento. }\end{array}$ \\
\hline 4 & $\begin{array}{l}\text { Dolbow y } \\
\text { Figoni, } 2015 \\
(28)\end{array}$ & $\begin{array}{l}\text { Estados } \\
\text { Unidos }\end{array}$ & $\begin{array}{l}10 \text { instalaciones pú- } \\
\text { blicas de acondicio- } \\
\text { namiento físico. }\end{array}$ & Ejercicio & $\begin{array}{l}\text { Lista de veri- } \\
\text { ficación adap- } \\
\text { tada de la ADA } \\
\text { utilizada por } \\
\text { Figoni et al. } \\
\text { (1998), con } 74 \\
\text { ítems, con la } \\
\text { secciones de } \\
\text { personas de- } \\
\text { pendientes de } \\
\text { sillas de rue- } \\
\text { das y más allá } \\
\text { de las pautas } \\
\text { exigidas. }\end{array}$ & $\begin{array}{l}\text { Accesibilidad física: escri- } \\
\text { torio de atención al clien- } \\
\text { te, fuentes de consumo de } \\
\text { agua, baños, ascensores, } \\
\text { caminos/ rutas, entradas } \\
\text { exteriores/puertas, rampas. } \\
\text { Accesibilidad al desplaza- } \\
\text { miento: estacionamiento. } \\
\text { Accesibilidad en imple- } \\
\text { mentación deportiva y } \\
\text { recreativa: equipamiento, } \\
\text { accesibilidad para y alrede- } \\
\text { dor del equipo de ejercicio. }\end{array}$ \\
\hline
\end{tabular}




\begin{tabular}{|c|c|c|c|c|c|c|}
\hline n. ${ }^{\circ}$ & Referencia & $\begin{array}{l}\text { País de } \\
\text { origen }\end{array}$ & $\begin{array}{c}\text { Número y } \\
\text { características } \\
\text { de la muestra } \\
\text { (instalaciones de } \\
\text { acondicionamiento) }\end{array}$ & $\begin{array}{l}\text { Tipo de } \\
\text { práctica }\end{array}$ & $\begin{array}{l}\text { Instrumento } \\
\text { de medición }\end{array}$ & $\begin{array}{c}\text { Identificación del } \\
\text { tipo y descripción de } \\
\text { accesibilidad al entorno } \\
\text { físico (dominios/ } \\
\text { secciones de evaluación } \\
\text { seleccionadas) }\end{array}$ \\
\hline 5 & $\begin{array}{l}\text { Pike et al., } \\
2008 \text { (29) }\end{array}$ & $\begin{array}{l}\text { Estados } \\
\text { Unidos }\end{array}$ & $\begin{array}{l}52 \text { instalaciones } \\
\text { acuáticas abiertas al } \\
\text { público en general. }\end{array}$ & $\begin{array}{l}\text { Actividad } \\
\text { física }\end{array}$ & $\begin{array}{l}\text { Encuesta mo- } \\
\text { dificada de } \\
9 \text { dominios } \\
\text { estructurales } \\
\text { y más de } 49 \\
\text { características } \\
\text { basada en una } \\
\text { revisión de } \\
\text { ADAAG y del su- } \\
\text { plemento. }\end{array}$ & $\begin{array}{l}\text { Accesibilidad física: mos- } \\
\text { trador de boletos, puerta/ } \\
\text { entrada, baño de hombres, } \\
\text { baño de mujeres, vestidor, } \\
\text { fuentes de agua potable, ca- } \\
\text { minos, entrada a la piscina. } \\
\text { Accesibilidad al desplaza- } \\
\text { miento: estacionamiento. }\end{array}$ \\
\hline 6 & $\begin{array}{l}\text { Cardinal y } \\
\text { Spaziani, } \\
2003(30)\end{array}$ & $\begin{array}{l}\text { Estados } \\
\text { Unidos }\end{array}$ & $\begin{array}{l}50 \text { instalaciones } \\
\text { de actividad física } \\
\text { abiertas para hom- } \\
\text { bres y mujeres. }\end{array}$ & $\begin{array}{l}\text { Actividad } \\
\text { física }\end{array}$ & $\begin{array}{l}\text { Lista de veri- } \\
\text { ficación modi- } \\
\text { ficada para la } \\
\text { evaluación de } \\
\text { la accesibili- } \\
\text { dad de las ins- } \\
\text { talaciones de } \\
\text { acondiciona- } \\
\text { miento físico } \\
\text { de Figoni et al., } \\
\text { con } 76 \text { ítems. }\end{array}$ & $\begin{array}{l}\text { Accesibilidad física: escri- } \\
\text { torio de servicio al cliente, } \\
\text { fuentes de agua potable, } \\
\text { baños/vestuarios, ascen- } \\
\text { sores, caminos/recorridos, } \\
\text { entradas/puertas exterio- } \\
\text { res, rampas. } \\
\text { Accesibilidad al desplaza- } \\
\text { miento: estacionamiento. } \\
\text { Accesibilidad en imple- } \\
\text { mentación deportiva y } \\
\text { recreativa: accesibilidad } \\
\text { para y alrededor del equi- } \\
\text { po de ejercicio. }\end{array}$ \\
\hline 7 & $\begin{array}{l}\text { Rimmer } \\
\text { et al., } \\
2005 \text { (31) }\end{array}$ & $\begin{array}{l}\text { Estados } \\
\text { Unidos }\end{array}$ & $\begin{array}{l}35 \text { instalaciones de } \\
\text { acondicionamiento } \\
\text { físico/clubes de salud }\end{array}$ & $\begin{array}{l}\text { Actividad } \\
\text { física } \\
\text { Ejercicio }\end{array}$ & $\begin{array}{l}\text { Versión modi- } \\
\text { ficada del Ins- } \\
\text { trumento de } \\
\text { Accesibilidad } \\
\text { para la Medi- } \\
\text { ción de Entor- } \\
\text { nos de Recrea- } \\
\text { ción y de Acon- } \\
\text { dicionamiento } \\
\text { Físico. }\end{array}$ & $\begin{array}{l}\text { Accesibilidad física: en- } \\
\text { torno construido. } \\
\text { Accesibilidad al desplaza- } \\
\text { miento: estacionamiento. } \\
\text { Accesibilidad en imple- } \\
\text { mentación deportiva y re- } \\
\text { creativa: equipamiento. }\end{array}$ \\
\hline
\end{tabular}

Consideraciones éticas. De acuerdo con el artículo 11 de la Resolución 8430 de 1993, esta investigación no presenta riesgo por ser de tipo documental, ya que no se realiza ninguna intervención o modificación intencionada de las variables biológicas, fisiológicas, psicológicas o sociales de los individuos (24). 


\section{Resultados}

e los siete estudios seleccionados, se analizaron las siguientes categorías: país de origen, número total y características de la muestra, clasificación del tipo de práctica y el tipo de accesibilidad al entorno físico e instrumentos de medición.

\section{País de origen}

Se identificaron tres países: Canadá, en la región de Ontario, Portugal, en la región de Oporto, y Estados Unidos. Este último con una amplia red de cobertura de examinación de instalaciones en doce de los cincuenta estados que conforman todo el país, los cuales incluyen Iowa (IA), Illinois (IL), Indiana (IN), Massachusetts (ма), Michigan (мI), Missouri (мо), Montana (мт), Ohio (он), Wisconsin (wI), Texas (тx), Hattiesburg Mississippi (мs) y Oregón (oR), y nueve de las diez regiones en las que se divide todo el país: Nueva Inglaterra, Noreste, Atlántico medio, Sureste, Grandes Lagos, Grandes Planicies, Montañas Rocosas, Suroeste y Noroeste (27-31). De estos la mayoría son de origen estadounidense (71.42\%). La importancia de resaltar el país de origen y la región radica en mostrar que en Norteamérica y Europa, especialmente en Estados Unidos, han sido pioneros en promover la investigación de accesibilidad para personas con discapacidad en instalaciones de acondicionamiento.

\section{Número total y características de la muestra (instalaciones de acondicionamiento físico)}

Se identificaron 429 instalaciones de acondicionamiento físico, con las siguientes características:

- Ubicación/región geográfica: 175 (40.8\%) en zona urbana, 131 (30.5\%) en zona suburbana, $0(0.0 \%)$ en zona rural y 123 (28.7\%) sin registro de ubicación $(25,27,31)$.

- Tipo de negocio: 35 (8.2\%) instalaciones con fines de lucro o privadas, 248 (57.8\%) instalaciones sin fines de lucro o públicas y 146 (34.0\%) sin registro de tipo de ubicación (26-28,31).

- Afiliación de las instalaciones: 220 (51.3\%) centros de acondicionamiento/clubes de salud, 37 (8.6\%) parques del distrito/centros comunitarios, 4 (0.9\%) centros de recreación, 54 (12.6\%) centros acuáticos, 11 (2.6\%) complejos/instalaciones deportivas, 14 (3.3\%) hospitales/centros de rehabilitación y 89 (20.7\%) universidades/colegios $(27,30,31)$.

- Fechas/cambios de accesibilidad: 30 (7.0\%) instalaciones antes de 1999, 10 (2.3\%) instalaciones después del 2000, 26 (6.1\%) instalaciones con cambios de accesibilidad menores a 10 años, 109 (25.4\%) instalaciones con construcciones antes del acta ADA entre 1990 y 1992 
y 109 (25.4\%) instalaciones con construcciones después del acta ADA entre 1990 y 1992 y 145 (33.8\%) sin registro de fechas/cambios $(25,27,29)$.

\section{Clasificación del tipo de práctica y el tipo de accesibilidad al entorno físico}

El tipo de práctica se definió bajo los conceptos de actividad física, ejercicio y deporte (15,32-35). El tipo de accesibilidad al entorno físico se enmarcó dentro de los lineamientos de Coldeportes para la presentación de resultados y discusión, con los conceptos de accesibilidad física, accesibilidad al desplazamiento y accesibilidad a la implementación deportiva y recreativa (15). En cinco estudios (71\%) se encontró que la actividad física es el tipo de práctica que más se realiza en las instalaciones de acondicionamiento físico $(25,27,29,31)$; seguida del ejercicio, incluido en cuatro estudios (57\%), y, por último, el deporte incluido en un estudio (14\%) (25-28,31). Por otra parte, en todos los estudios se examinó la accesibilidad al entorno físico $(25,27,28,31)$; mientras que la accesibilidad al desplazamiento fue examinada en seis estudios (86\%), y la accesibilidad a la implementación deportiva y recreativa, en cinco estudios (71\%) (25,27-31).

\section{Instrumentos de medición}

Se identificaron cuatro instrumentos con medidas de resultados similares de puntuación media en porcentajes en todos los estudios, excepto en el estudio de Rimmer et al., ya que la presentación de los resultados se encuentra por ítems de los dominios sin ningún valor numérico porcentual exacto $(26,31)$. A continuación, se presentan los instrumentos identificados: 1) Instrumento de Accesibilidad para la Medición de Entornos de Recreación y de Acondicionamiento Físico (25,27,31); 2) Encuesta basada en las Guías de accesibilidad del acta ADA y del suplemento (29); 3) Lista de Verificación de Cumplimiento del Acta ADA, utilizada anteriormente por Figoni et al. $(28,30)$; y, 4) Creación de la lista de verificación basada en las normas y la legislación portuguesa y europea, con elementos que deben cumplir las instalaciones deportivas para la accesibilidad y la seguridad de las personas con movilidad reducida (26).

Basándose en el estudio de revisión sistemática de Calder et al., los resultados se interpretaron en la tabla 2 de manera porcentual por tipo de accesibilidad al entorno físico (accesibilidad física, accesibilidad al desplazamiento y accesibilidad a la implementación deportiva y recreativa), según cada estudio incluido, identificado con el tipo de instalación de acondicionamiento, donde $0 \%$ es la accesibilidad mínima y el 100\% es la accesibilidad máxima (36). 
Tabla 2. Puntuaciones por tipo de dominio o secciones de accesibilidad

\begin{tabular}{|c|c|c|c|c|c|c|}
\hline $\begin{array}{l}\text { Tipo de instalaciones } \\
\text { de acondicionamiento }\end{array}$ & $\begin{array}{c}\text { Instalacio- } \\
\text { nes recreati- } \\
\text { vas y centros } \\
\text { de acondi- } \\
\text { cionamiento } \\
\text { físico (25) }\end{array}$ & $\begin{array}{c}\text { Complejos/ } \\
\text { instalacio- } \\
\text { nes deporti- } \\
\text { vas públicas } \\
\text { (26) }\end{array}$ & $\begin{array}{l}\text { Centros de } \\
\text { acondicio- } \\
\text { namiento } \\
\text { físico (27) }\end{array}$ & $\begin{array}{l}\text { Instala- } \\
\text { ciones pú- } \\
\text { blicas de } \\
\text { acondicio- } \\
\text { namiento } \\
\text { físico (28) } \\
\end{array}$ & $\begin{array}{c}\text { Instala- } \\
\text { ciones } \\
\text { acuáticas } \\
\quad(29)\end{array}$ & $\begin{array}{l}\text { Instalacio- } \\
\text { nes de acti- } \\
\text { vidad física } \\
\quad(30)\end{array}$ \\
\hline $\begin{array}{l}\text { Dominios/secciones } \\
\text { de accesibilidad }\end{array}$ & $\begin{array}{c}\text { Media } \\
(\%)\end{array}$ & $\begin{array}{c}\text { Media } \\
\text { (\%) }\end{array}$ & $\begin{array}{c}\text { Media } \\
\text { (\%) }\end{array}$ & $\begin{array}{c}\text { Media } \\
\text { (\%) }\end{array}$ & $\begin{array}{c}\text { Media } \\
\text { (\%) }\end{array}$ & $\begin{array}{c}\text { Media } \\
\text { (\%) }\end{array}$ \\
\hline $\begin{array}{l}\text { Áreas de entrada (rutas de } \\
\text { acceso, puertas de entrada y } \\
\text { recepción) }\end{array}$ & 51.60 & - & 50.62 & - & - & - \\
\hline Puertas exteriores/entrada & - & - & - & 50.00 & 95.00 & 90.00 \\
\hline Camino/pasillos & - & - & - & 60.00 & 87.00 & 58.00 \\
\hline Rampas & - & - & - & 70.00 & - & 83.00 \\
\hline Atención al cliente/taquilla & - & - & - & 50.00 & 69.90 & 37.00 \\
\hline Vestuarios/duchas/baños & - & 59.59 & 53.03 & - & 63.90 & - \\
\hline Vestuarios & 42.20 & 54.55 & - & - & 55.70 & 44.00 \\
\hline $\begin{array}{l}\text { Spas (tinas de hidromasaje, } \\
\text { bañeras, saunas, salas de vapor) }\end{array}$ & - & - & 28.53 & - & - & - \\
\hline Baños & 31.10 & - & 44.55 & 20.00 & - & - \\
\hline Ascensores & 49.00 & - & - & 100.00 & - & 83.00 \\
\hline Fuentes de agua potable & - & - & 82.26 & 70.00 & 70.20 & 55.00 \\
\hline Teléfonos & - & - & 35.00 & - & - & 88.00 \\
\hline $\begin{array}{l}\text { Edificios o establecimientos en } \\
\text { general }\end{array}$ & - & 36.22 & - & - & - & - \\
\hline Estacionamiento & 54.50 & - & 66.94 & 80.00 & 78.20 & 56.00 \\
\hline Equipamiento & 47.70 & - & 53.16 & - & - & 8.00 \\
\hline Equipo adaptado especializado & - & - & - & 20.00 & - & - \\
\hline Accesibilidad de equipos & - & - & - & 0.00 & - & - \\
\hline Piscina & 37.00 & - & 47.43 & - & - & - \\
\hline Requisitos de entrada a piscina & - & - & - & - & 48.30 & - \\
\hline Información y señalización & - & - & 47.04 & - & - & - \\
\hline Políticas & 47.10 & - & 49.71 & - & - & - \\
\hline Formación y apoyo profesional & 47.90 & - & 62.68 & 0.00 & - & - \\
\hline Programas (actividades) & 63.10 & - & 71.66 & - & - & - \\
\hline ADAAG general & - & - & - & - & 69.90 & - \\
\hline General incluyendo suplemento & - & - & - & - & 65.80 & - \\
\hline
\end{tabular}

Azul: accesibilidad física; naranja: accesibilidad al desplazamiento; gris: accesibilidad a la implementación deportiva y recreativa; amarillo: accesibilidad (otros). 
En la figura 2 se observan los resultados de cada estudio por tipo de accesibilidad al entorno físico. La más alta puntuación media en porcentaje es la accesibilidad física (57.01\%), relacionada con los dominios del entorno físico, seguido por una escasa diferencia de la accesibilidad al desplazamiento (55.94\%), relacionada con el dominio de estacionamiento. Los resultados de la presente investigación encontrados en términos de accesibilidad física y accesibilidad al desplazamiento son cercanos; solamente se observó una diferencia menor al $2 \%$ de una media de $56.48 \%$ entre ambos. La puntuación media de la categoría “Otros” se encuentra entre los tipos de accesibilidad más baja (27.64\%), y se asocia con los dominios de piscina, requisitos de entrada a piscina, información y señalización, políticas de la instalación, formación y apoyo profesional y programas (actividades). Por último, se encuentra la accesibilidad a la implementación deportiva y recreativa con la puntuación considerablemente más baja (19.81\%), relacionada con los dominios equipamiento/equipamiento deportivo, equipo adaptado especializado y accesibilidad de equipos.

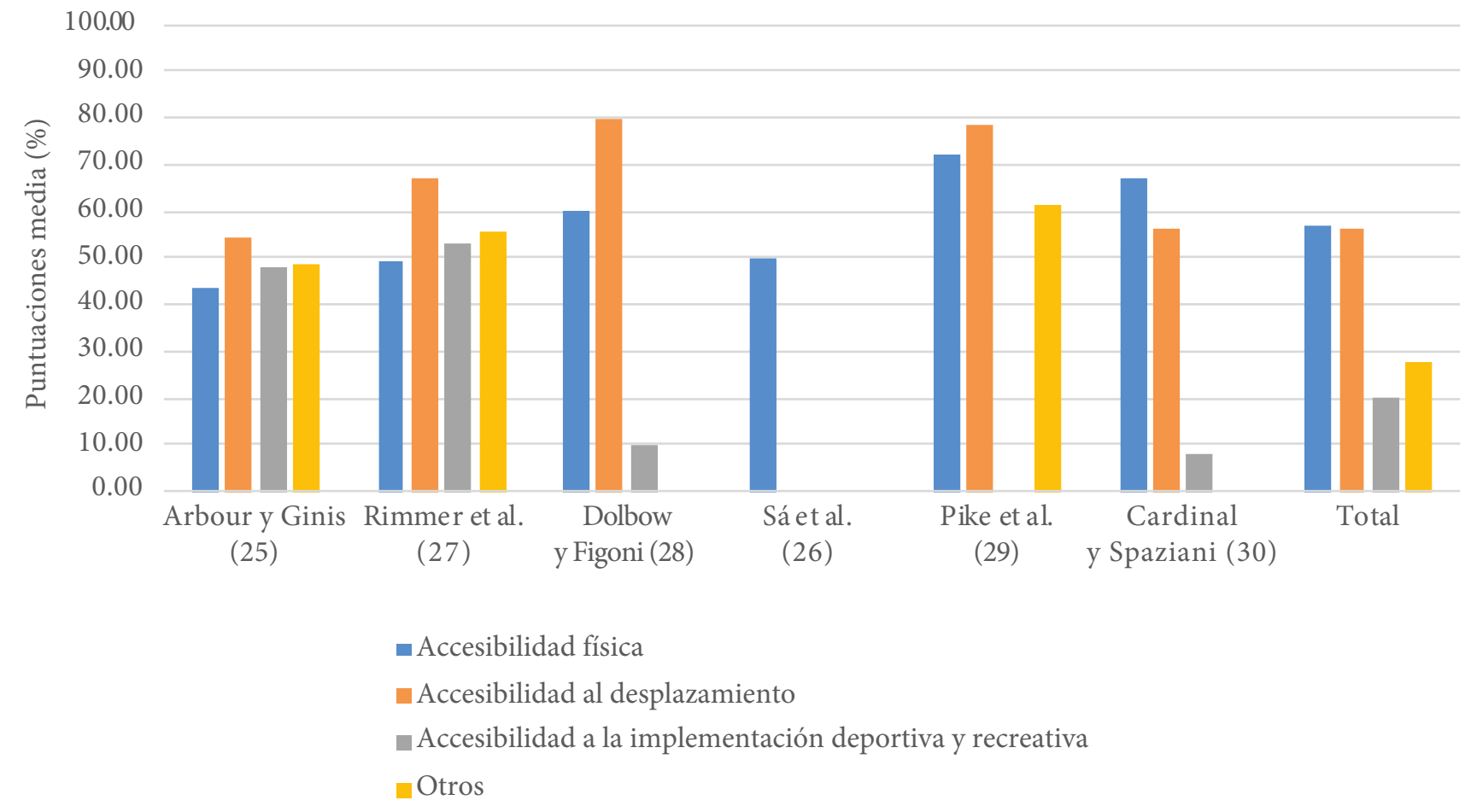

Figura 2. Puntuaciones por tipo de accesibilidad al entorno físico

\section{Discusión}

os siete estudios incluidos en esta revisión dan cuenta del escaso número de publicacio-
nes en revistas académicas sobre este tema. Desde el 2003 ya se conocían normas que 
revolucionaron la concepción internacional de accesibilidad, y muchas instalaciones lograron adaptaciones al entorno físico (11). Además, cabe resaltar que esta revisión se enfoca exclusivamente en la accesibilidad al entorno físico bajo los conceptos de los lineamientos de Coldeportes, motivo por el cual se toma esta temática desde el punto de vista internacional, para comenzar en Colombia futuras investigaciones, proyectos e intervenciones relacionadas (15). Dado que la mayoría de los estudios son de origen estadounidense, esto revela la gran necesidad en Latinoamérica y, fundamentalmente, en Colombia de producir investigación sobre este tema de interés, ya que solamente en Puerto Rico se ha identificado una tesis que abarcó este tema investigativo (37). Las características de las instalaciones de acondicionamiento evidenciadas muestran el amplio alcance que los lineamientos de Coldeportes deberían tener en cuenta en la toma de decisiones de accesibilidad al entorno físico local, departamental o nacional, y en los procesos de caracterización de centros de acondicionamiento físico, gimnasios, piscinas, escenarios recreativos y deportivos $(15,36)$.

La actividad física y el ejercicio son las prácticas más comunes en las instalaciones de acondicionamiento identificadas, que abarcan áreas de gimnasio, áreas o salones de ejercicios grupales, áreas de cardio, áreas de entrenamiento con pesas e instalaciones acuáticas $(15,32,33,38)$. La práctica del deporte es la que menos se realiza en las instalaciones/complejos deportivos ya identificados $(34,35)$. La evidencia describe que este problema puede darse por la presencia de algunas barreras ambientales, como la carencia de instalaciones deportivas, el transporte y las dificultades para acceder a la participación deportiva para personas con discapacidad (39). Sin embargo, los comités de deporte paralímpico, a través de los juegos paralímpicos, han permitido mostrar las habilidades y las aptitudes de las personas con discapacidad, garantizando derechos como la integración, la igualdad de oportunidades y la accesibilidad al entorno construido, al crear una mayor conciencia de las oportunidades que se deben concebir para esta población en cuanto a la participación deportiva (40). Todo esto se traduce en promoción de la salud, protección y velación de los derechos de las personas con discapacidad y la integración social a través del deporte (40). Un estudio demostró la relación de la accesibilidad de las instalaciones deportivas con el aumento de la actividad física en adultos coreanos, al referir que es crucial tener en cuenta la accesibilidad de las instalaciones deportivas cuando se promueve un entorno propicio para la actividad física y en el diseño de programas para mejorar la actividad física (41).

Los instrumentos de medición identificados en Estados Unidos (Accessibility Instruments Measuring Fitness and Recreation Environments, las guías de accesibilidad de la ADA y la lista de verificación de utilizada anteriormente por Figoni et al. están regidas por el título III del Acta ADA sobre los estándares de diseño accesible en instalaciones públicas y comerciales (11,42-45). Por otro lado, la lista de verificación desarrollada por Sá. et al. está basado en la ley portuguesa 38 de 2004 sobre la prevención, empoderamiento, participación y rehabilitación de las personas con discapacidad (26). El Accessibility Instrument Measuring Fitness and Recreation Environments fue desarrollado y validado colectivamente por un análisis de modelo rasch, el 
cual demostró que es adecuado/bueno en propiedades psicométricas de consistencia interna ( $r=0.70-0.90)$ y de confiabilidad test-retest con una variación de 0.70-0.97 (46).

Mediante una revisión sistemática, se identificaron siete instrumentos en total entre los años 1991 y 2010, de los cuales tres de ellos ya han sido identificados por esta revisión (47) mientras que los cuatro instrumentos restantes son: 1. Community Health Environment Checklist - Mobility, 2. Removing Barriers to Health Clubs and Fitness Facilities, 3. Health Empowerment Zone Environmental Checklist: Fitness Centre Survey y 4. Community Health Environment Checklist - Exercise Facilities (47). En Colombia, la falta de identificación de los beneficios y del impacto positivo de los lineamientos de Coldeportes incentivó la creación de una herramienta de evaluación, la cual busca monitorizar la implementación de actividades o programas enfocados en el fomento de la práctica de recreación, actividad física y deporte en personas con discapacidad (48). Pese a que este es un primer paso para mejorar los métodos actuales dirigidos a fortalecer los datos existentes, y para obtener una visión de impacto de los planes, proyectos y programas para implementar, esta herramienta no presenta indicadores robustos que reflejen el contenido específico del lineamiento de accesibilidad al entorno físico (48).

Los resultados encontrados en términos de accesibilidad física y accesibilidad al desplazamiento son similares a los de Calder et al., puesto que los dominios tomados para la clasificación de accesibilidad al entorno físico no poseen una alta accesibilidad y se encuentran en el rango de accesibilidad entre el $33 \%$ y el $68 \%$, aproximadamente (36). En la accesibilidad al desplazamiento, nuestro estudio es limitado, ya que por la literatura encontrada solo se pudo tener en cuenta el dominio único de estacionamiento, que en realidad abarcaría una parte de este concepto, pues, a pesar de que investigaciones describen la falta de recursos de transportación y dificultades para la consecución de transporte, ninguno de los estudios incluidos en esta revisión tomó en cuenta este aspecto de interés definido por los lineamientos de Coldeportes, donde resalta la necesidad de alianzas estratégicas con entidades públicas o privadas para la oferta y la transportación, y así facilitar el desplazamiento $(15,49,50)$.

Por otro lado, los resultados muestran que la accesibilidad a la implementación deportiva y recreativa es considerablemente más baja, junto con la menor examinación dentro de la muestra de estudios incluidos. Ello evidencia, aún más, la necesidad de investigar en este aspecto, puesto que se han identificado tres principales barreras: espacio entre el equipo para el acceso de silla de ruedas, mantenimiento deficiente del equipo y falta de equipo adaptable o accesible (49). En la práctica de actividad física y ejercicio, estudios han definido las áreas de entrenamiento y el uso de equipos, accesibilidad para y alrededor del equipo (máquinas y estaciones de ejercicio), dotación de personal, equipos especializados o equipos adaptados como el scifrт máquina de ejercicio y ergómetro de extremidades superiores $(28,38)$. En el caso del deporte, la implementación debe ser adecuada o adaptada si así lo requiere, según las necesidades específicas de cada deporte en limitaciones físicas (ajedrez, atletismo, baloncesto en silla de ruedas, billar, 
ciclismo, levantamiento de pesas, natación, tenis de campo en silla de ruedas, tenis de mesa, tiro deportivo) definidas por el deporte paralímpico colombiano $(51,52)$.

Finalmente, la sección de accesibilidad “Otros” (piscina, requisitos de entrada a piscina, información y señalización políticas de la instalación, formación y apoyo profesional, programas-actividades y más allá de los requisitos), también se encuentra entre los tipos de accesibilidad más baja, lo cual concuerda con los resultados encontrados por Rimmer et al., en los cuales el $36 \%$ de los participantes $(n=83)$ indicaron que el personal no tenía la suficiente formación para lograr satisfacer sus necesidades (53).

Las limitaciones de estudio atañen a la pequeña muestra de estudios incluidos, debido a la escasa literatura existente. Otra limitación es el abordaje de un segmento del concepto integral de accesibilidad, pues este es amplio y tiene un mayor impacto cuando se aplica la accesibilidad en todas sus dimensiones (por ejemplo, actitud e imaginarios sociales, información, calidad del servicio de los programas, políticas, formación y entrenamiento del personal, entre otros), y no solo desde el entorno físico. Futuros estudios deben enfocarse en la investigación y publicación académica de accesibilidad y accesibilidad al entorno físico. Se requieren estudios que diseñen y validen un instrumento apto para realizar la evaluación de accesibilidad a las instalaciones de acondicionamiento físico en Colombia, teniendo en cuenta las perspectivas teóricas del modelo social de discapacidad y de la investigación-acción participativa, las cuales permitirán obtener un concepto más amplio y claro de las condiciones en las cuales se encuentran estas instalaciones, logrando la modificación o transición de las áreas críticas con los siguientes pasos: 1) evaluación de accesibilidad, 2) revisión de la accesibilidad, 3) planes de transición de accesibilidad y 4) desarrollo e implementación de una base de datos de soluciones (54).

En conclusión, se evidencia que las instalaciones de acondicionamiento todavía no son del todo accesibles para personas que se encuentran en condición de discapacidad. Se mostraron algunos avances en cuanto a la accesibilidad física, pero hace falta mayor investigación en cuestión de accesibilidad al desplazamiento y primordialmente en accesibilidad en implementación deportiva y recreativa. La accesibilidad de las personas con discapacidad a los clubes de salud y acondicionamiento físico no es una cuestión política; más bien, es un tema de justicia social. Si queremos trabajar por crear un país en el cual todos los individuos sean valorados, entonces debemos crear entornos donde todos puedan beneficiarse de los bienes y servicios, independientemente de si se tiene o no una discapacidad. Tenemos que alentary promover más investigación sobre accesibilidad para poder crear el tipo de comunidades con conciencia social donde todos podamos prosperar. 


\section{Contribución de los autores}

Cada uno de los autores declara que ha realizado la planeación, la concepción y diseño, la adquisición, el análisis e interpretación de los datos e información, la revisión del contenido intelectual importante y la aprobación del presente artículo. Una versión preliminar fue presentada en el Quinto Encuentro Nacional de Semilleros de Investigación de Ciencias de la Salud, en formato de póster electrónico en la ciudad de Bogotá, el día 21 de agosto de 2019.

\section{Descargos de responsabilidad}

F sta investigación no recibió ninguna subvención específica de agencias de financiamiento Een los sectores público, comercial o sin fines de lucro.

\section{Conflicto de intereses}

Ninguno declarado.

\section{Referencias}

1. Organización Mundial de la Salud, Banco Mundial. Informe mundial sobre la discapacidad [internet]. 2011 [citado 2019 ene 25]. Disponible en: https://www.who.int/disabilities/ world_report/2011/summary_es.pdf

2. Correa L, Castro M. Discapacidad e inclusión social en Colombia: informe alternativo de la Fundación Saldarriaga Concha al Comité de Naciones Unidas sobre los derechos de las personas con discapacidad. Bogotá: Editorial Fundación Saldarriaga Concha; 2016.

3. Lugo Agudelo LH, Seijas V. La discapacidad en Colombia: una mirada global. Rev Col Med Fís Rehab. 2012;22(2):164-79.

4. Departamento Administrativo Nacional de Estadística (DANE). Censo Nacional de Población y Vivienda-cNPv 2018 [internet]. 2018 [citado 2019 feb 3]. Disponible en: https:// sitios.dane.gov.co/cnpv-presentacion/src/\#cuantos00

5. Ministerio de Salud y Protección Social. Sala situacional de las personas con discapacidad (PCD) [internet]. 2018 [citado 2019 feb 3]. Disponible en: https://www.minsalud.gov.co/ sites/rid/Lists/BibliotecaDigital/RIDE/DE/PS/sala-situacional-discapacidad-junio-2018.pdf

6. Organización Mundial de la Salud. Discapacidad y salud [internet]. 2018 [citado 2019 feb 8]. Disponible en: https://www.who.int/es/news-room/fact-sheets/detail/disability-and-health

7. Lakowski T, Long T. Proceedings: physical activity and sport for people with disabilities. Washington, DC: Georgetown University Center for Child and Human Development; 2011. 
8. Gross J, Kroll T, Morris J. Accessibility of fitness centres for people with disabilities in a region in North East Scotland. Public Health. 2013;127(8):782-4. https://doi.org/10.1016/j. puhe.2013.04.030

9. Casajús JA, Vicente-Rodríguez G. Ejercicio físico y salud en poblaciones especiales. Exernet. Madrid: Consejo Superior de Deportes \& Presidencia de Gobierno; 2011.

10. National Center on Health, Physical Activity and Disability (NCHPAD). Physical activity guidelines for adults with disabilities: NCHPAD-building inclusive communities [internet]. [citado 2019 mar 10]. Disponible en: https://www.nchpad.org/618/2576/ Physical Activity Guidelines for Adults with Disabilities

11. United States Department of Justice Civil Rights Division. Americans with Disabilities Act 2010 Standards for Public Accommodations and Commercial Facilities: Title III. 2010 sep 15.

12. Departamento Nacional de Estadística (DANE). Informe al Congreso 2007-2008: Censo de Escenarios Deportivos y Recreativos [internet]. 2008 [citado 2019 abr 17]. Disponible en: https://www.dane.gov.co/files/control_participacion/rendicion_cuentas/informe_congreso_07.pdf

13. Instituto Colombiano del Deporte (Coldeportes), Comité Paralímpico Colombiano (CPC), Asociación Colombiana de Universidades (Ascun), Federación Colombiana de Organizaciones de Personas con Discapacidad Física (Fecodif). Guía de diseño accesible y universal [internet]. 2009 [citado 2019 abr 23]. Disponible en: http://www.keroul.qc.ca/ DATA/PRATIQUEDOCUMENT/90_fr.pdf

14. Departamento Nacional de Planeación (DNP). Norma Técnica Colombiana de Accesibilidad NTC 6047 de 2013 [internet]. 2013 [citado 2019 abr 24]. Disponible en: https://www.dnp.gov. co/programa-nacional-del-servicio-al-ciudadano/Herramientas-Servicio/Soluciones\%20 para\%20la\%20inclusion\%20social/Paginas/Canal-Presencial.aspx

15. Instituto Colombiano del Deporte (Coldeportes). Lineamientos para el fomento y desarrollo de la inclusión de la población con discapacidad en educación física, recreación, actividad física y deporte [internet]. 2013 [citado 2019 abr 24]. Disponible en: http:// www.coldeportes.gov.co/fomento_desarrollo/lineamientos_poblacion_discapacidad/ lineamientos_fomento_desarrollo_64513

16. Guirao Goris JA. Utilidad y tipos de revisión de literatura. Rev Enferm. 2015;9(2). https:// doi.org/10.4321/S1988-348X2015000200002

17. Whittemore R. Combining evidence in nursing research: methods and implications. Nurs Res. 2005;54(1):56-62. https://doi.org/10.1097/00006199-200501000-00008

18. Whittemore R, Knafl K. The integrative review: updated methodology. J Adv Nurs. 2005;52(5):546-53. https://doi.org/10.1111/j.1365-2648.2005.03621.x

19. Smith MC, Stullenbarger E. A prototype for integrative review and meta-analysis of nursing research. J Adv Nurs. 1991;16(11):1272-83. https://doi.org/10.1111/j.1365-2648.1991. tb01554.x

20. Resolución 00583 de 2018/28 de febrero, por la cual se implementa la certificación de discapacidad y el registro de localización y caracterización de personas con discapacidad [internet]. Ministerio de Salud y Protección Social. Disponible en: https://www. minsalud.gov.co/sites/rid/Lists/BibliotecaDigital/RIDE/DE/DIJ/Resolucion-583-de-2018.pdf 
21. Cooke A, Smith D, Booth A. Beyond PICo: the SPIDER tool for qualitative evidence synthesis. Qual Health Res. 2012;22(10):1435-43. https://doi.org/10.1177/1049732312452938

22. Mesquita AC, Carvalho EC de, Mesquita AC, Carvalho EC de. Therapeutic listening as a health intervention strategy: an integrative review. Rev da Esc Enferm usp. 2014;48(6):112736. https://doi.org/10.1590/S0080-623420140000700022

23. Kiersma ME, Plake KS, Darbishire PL. Patient safety instruction in US health professions education. Am J Pharm Educ. 2011;75(8):162. https://doi.org/10.5688/ajpe758162

24. Resolución 8430 de 1993/4 de octubre, por la cual se establecen las normas científicas, técnicas y administrativas para la investigación en salud [internet]. Ministerio de Salud. Disponible en: https://www.minsalud.gov.co/sites/rid/Lists/BibliotecaDigital/RIDE/DE/ DIJ/RESOLUCION-8430-DE-1993.PDF

25. Arbour-Nicitopoulos KP, Ginis KAM. Universal accessibility of "accessible" fitness and recreational facilities for persons with mobility disabilities. Adapt Phys Activ Q. 2011;28(1):1-15. https://doi.org/10.1123/apaq.28.1.1

26. Sá MM, Azevedo R, Martins MC, Machado O, Tavares J. Accessibility of sports facilities for persons with reduced mobility and assessment of their motivation for practice. Work. 2012;41(Suppl 1):2017-23. https://doi.org/10.3233/WOR-2012-0425-2017

27. Rimmer JH, Padalabalanarayanan S, Malone LA, Mehta T. Fitness facilities still lack accessibility for people with disabilities. Disabil Health J. 2017;10(2):214-21. https://doi. org/10.1016/j.dhjo.2016.12.011

28. Dolbow DR, Figoni SF. Accommodation of wheelchair-reliant individuals by community fitness facilities. Spinal Cord. 2015;53(7):515-9. https://doi.org/10.1038/sc.2015.26

29. Pike H, Walker J, Collins J, Hodges J. An investigation of ADA compliance of aquatic facilities in the North Texas area. Am J Health Promot. 2008;23(2):139-46. https://doi. org/10.4278/ajhp.07041336

30. Cardinal BJ, Spaziani MD. ADA compliance and the accessibility of physical activity facilities in Western Oregon. Am J Health Promot. 2003;17(3):197-201. https://doi. org/10.4278/0890-1171-17.3.197

31. Rimmer JH, Riley B, Wang E, Rauworth A. Accessibility of health clubs for people with mobility disabilities and visual impairments. Am J Public Health. 2005;95(11):2022-8. https://doi.org/10.2105/AJPH.2004.051870

32. Pérez Samaniego V, Devís Devís J. La promoción de la actividad física relacionada con la salud: la perspectiva de proceso y de resultado. Rev Int Med Cienc Act Fis Deporte. 2003;3(10):69-74.

33. American College of Sports Medicine. Acsm's guidelines for exercise testing and prescription. Filadelfia: Lippincott Williams \& Wilkins; 2013.

34. Ley 181 de 1995/18 de enero, por la cual se dictan disposiciones para el fomento del deporte, la recreación, el aprovechamiento del tiempo libre y la Educación Física y se crea el Sistema Nacional del Deporte [internet]. Congreso de la República de Colombia. Disponible en: https://www.mindeporte.gov.co/recursos_user/2019/Juridica/ Normograma/Leyes/Ley-181-de-1995.pdf 
35. Ley 1946 de 2019/4 de enero, por medio del cual se modifica la ley 582 de 2000 y se dictan otras disposiciones [internet]. Congreso de Colombia. Disponible en: https://dapre.presidencia.gov.co/normativa/normativa/LEY\%201946\%20DEL\%204\%20DE\%20ENERO\%20 DE\%202019.pdf

36. Calder A, Sole G, Mulligan H. The accessibility of fitness centers for people with disabilities: A systematic review. Disabil Health J. 2018;11(4):525-36. https://doi.org/10.1016/j. dhjo.2018.04.002

37. Morales Jiménez CN, Mercado Quiñones V, Cruz Salgado JA. Evaluación de la accesibilidad para personas con discapacidades a los centros de acondicionamiento físico en el área metropolitana de Puerto Rico. San José: Universidad de Puerto Rico; 2012.

38. Petersen JC, Piletic CK. Facility accessibility: opening the doors to all. J Phys Educ Recreat Dance. 2006;77(5):38-44. https://doi.org/10.1080/07303084.2006.10597876

39. Jaarsma EA, Dijkstra PU, Geertzen JHB, Dekker R. Barriers to and facilitators of sports participation for people with physical disabilities: a systematic review. Scan J Med Sci Sports. 2014;24(6):871-81. https://doi.org/10.1111/sms.12218

40. Blauwet C, Willick SE. The paralympic movement: using sports to promote health, disability rights, and social integration for athletes with disabilities. PM\&R. 2012;4(11):8516. https://doi.org/10.1016/j.pmrj.2012.08.015

41. Lee SA, Ju YJ, Lee JE, Hyun IS, Nam JY, Han K-T, et al. The relationship between sports facility accessibility and physical activity among Korean adults. BMC Public Health. 2016;16(1):893. https://doi.org/10.1186/s12889-016-3574-z

42. National Center on Health, Physical Activity and Disability (NCHPAD). AIMFREE manuals NCHPAD building healthy inclusive communities [internet]. 2019 [citado 2019 abr 20]. Disponible en: https://www.nchpad.org/426/2254/AIMFREE Manuals

43. U.S. Architectural and Transportation Barriers Compliance Board (Access Board). Americans with Disabilities Act (ADA) accessibility guidelines for buildings and facilities [internet]. 2002 [citado 2019 may 4]. Disponible en: https://www.access-board.gov/ attachments/article/1350/adaag.pdf

44. Institute for Human Center Design, ADA National Network. ADA checklist for existing facilities [internet]. 2016 [citado 2019 may 5]. Disponible en: https://www.adachecklist. org/doc/fullchecklist/ada-checklist.pdf

45. Figoni SF, McClain L, Bell AA, Degnan JM, Norbury NE, Rettele RR. Accessibility of physical fitness facilities in the Kansas City metropolitan area. Top Spinal Cord Inj Rehabil. 1998;3(3):66-78.

46. Rimmer J, Riley B, Wang E, Rauworth A. Development and validation of AImfreE: Accessibility instruments measuring fitness and recreation environments. Disabil Rehabil. 2004;26(18):1087-95. https://doi.org/10.1080/09638280410001711432

47. Calder AM, Mulligan HF. Measurement properties of instruments that assess inclusive access to fitness and recreational sports centers: A systematic review. Disabil Health J. 2014;7(1):26-35. https://doi.org/10.1016/j.dhjo.2013.06.003

48. Almonacid Vélez JG. Herramienta de evaluación para programas de inclusión en actividad física, recreación y deporte para personas con discapacidad en Colombia [tesis 
de maestría]. Bogotá D.C.: Escuela de Medicina y Ciencias de la Salud, Universidad del Rosario; 2016.

49. Rimmer JH, Riley B, Wang E, Rauworth A, Jurkowski J. Physical activity participation among persons with disabilities: barriers and facilitators. Am J Prev Med. 2004;26(5):41925. https://doi.org/10.1016/j.amepre.2004.02.002

50. Serrano Ruiz CP, Ramírez Ramírez C, Abril Miranda JP, Ramón Camargo LV, Guerra Urquijo LY, Clavijo González N. Barreras contextuales para la participación de las personas con discapacidad física: discapacidad y barreras contextuales. Salud UIS. 2013;45(1):41-51.

51. Sport England. Accessible sports facilities formerly known as access for disabled people updated 2010 guidance [internet]. 2010 [citado 2019 may 5]. Disponible en: https://www. sportengland.org/media/4508/accessible-sports-facilities-2010.pdf

52. Comité Paralímpico Colombiano (сPC). Tabla de deportes paralímpicos Sistema Nacional de Deporte Paralímpico [internet]. 2019 [citado 2019 may 7]. Disponible en: https://comiteparalimpicocolombiano.org/item/categoria_cms/1/articulo/10/

53. Rimmer JH, Wang E, Smith D. Barriers associated with exercise and community access for individuals with stroke. J Rehabil Res Dev. 2008;45(2):315-22. https://doi.org/10.1682/ JRRD.2007.02.0042

54. Riley BB, Rimmer JH, Wang E, Schiller WJ. A conceptual framework for improving the accessibility of fitness and recreation facilities for people with disabilities. J Phys Act Health. 2008;5(1):158-68. https://doi.org/10.1123/jpah.5.1.158 